\title{
Representing Attitudes as Sets of Frames
}

\author{
Pål Johan From and Jan Tommy Gravdahl
}

\begin{abstract}
A general framework for representing continuous sets of frames with the unit quaternion representation is presented. The determination and control of the attitude of a rigid body is important in a wide range of applications and has been given much attention in the control community. Not always, however, must the desired attitude be restricted to one given orientation, but can be given as a discrete or continuous set of orientations subject to some restriction. An attitude can be represented by the four-parameter unit quaternion without the presence of singularities. It is shown how continuous sets of frames can be described by the unit quaternion representation. It is also shown how this set can be reorientated into an arbitrary coordinate system by the quaternion product. Some work is done on finding the attitude that is closest to the desired orientation when the desired orientation is out of reach due to some restriction on the allowed orientations or rotations.
\end{abstract}

\section{INTRODUCTION}

The attitude control problem of a rigid body is given much attention in the control community, and its applications range from attitude control of aircraft, spacecraft and satellites [1], [2] to rigid bodies held by robotic manipulators [3], [4]. A thorough discussion on the attitude control problem is given in [5], where global stability is shown for a variety of control laws using the unit quaternion representation in a Lyapunov function.

The unit quaternion group allows orientation and rotation to be represented globally without singularities. One problem of the unit quaternion group is that it is not as easy to visualise as the Euler angles. Many methods have been developed to help visualising quaternions and the relationship between quaternions and three-dimensional rotations. A good introduction on how to visualise quaternions can be found in [6] and [7]. [6] also gives a thorough presentation of quaternion curves, surfaces and volumes. Of special interest is the work presented on quaternion volumes, where it is shown that a continuous set of frames can be represented by a quaternion and a set of intuitive restrictions in Euler angle representation. The theory of quaternion volumes closely relates to orientation maps. Several techniques that can be used to visualise orientations are discussed in [8].

In this paper, the work on quaternion volumes is taken one step further, and a schematic approach on how to represent sets of frames is presented. It is shown how this set can be visualised by a set of points in the unit sphere, and how this set relate to the corresponding quaternion volume. It is also shown how this set can be reorientated so that it is defined with respect to some other reference frame. A test to verify if

The authors are with Department of Engineering Cybernetics, Norwegian University of Science and Technology, 7491 Trondheim, Norway. fromeitk. ntnu. no and tommy.gravdahleitk. ntnu.no a quaternion satisfies the restrictions given by the quaternion volume is also presented.

This paper also addresses the problem of how the unit quaternion group can be utilised to find the attitude that is closest to some given orientation when rotations about one axis only are allowed. This work is similar to the results found in [3], [5] and [6] in how the orientation error is presented, but goes one step further in also finding the closest orientation.

\section{REPRESENTING ROTATIONS}

Most of the fundamental principles of rotation were presented in two papers by Leonhard Euler in 1775 [8]. The first paper shows that any rotation can be accomplished by a sequence of three rotations about the coordinate axes. In the second paper, Euler states that any orientation can be represented by a rotation of some angle $\phi$ about some fixed axis $\boldsymbol{n}$. He also shows that the composition of two rotations is itself a rotation.

\section{A. The Unit Quaternion}

The unit quaternion representation closely relates to the results presented in Euler's second paper. A good introduction to quaternions is found in [7]. Any positive rotation $\phi$ about a fixed unit vector $\boldsymbol{n}$ can be represented by the four-tuple

$$
Q=\left[\begin{array}{c}
q_{0} \\
\boldsymbol{q}
\end{array}\right],
$$

where $q_{0} \in \mathbb{R}$ is known as the scalar part and $\boldsymbol{q} \in \mathbb{R}^{3}$ as the vector part. $Q(\phi, \boldsymbol{n})$ is written in terms of $\phi$ and $\boldsymbol{n}$ by

$$
q_{0}=\cos \left(\frac{\phi}{2}\right), \quad \boldsymbol{q}=\sin \left(\frac{\phi}{2}\right) \boldsymbol{n} .
$$

$Q$ is a quaternion of unit length and denoted a unit quaternion. Henceforth, all quaternions have unit length if not other is stated. A multiplication of two quaternions is given by a quaternion product and is written in vector algebra notations as

$$
P * Q=\left[\begin{array}{c}
p_{0} q_{0}-\boldsymbol{p} \cdot \boldsymbol{q} \\
p_{0} \boldsymbol{q}+q_{0} \boldsymbol{p}+\boldsymbol{p} \times \boldsymbol{q}
\end{array}\right] .
$$

The cross product implies that quaternion multiplication is not commutative, as expected. Let $P=\left[\begin{array}{llll}p_{0} & p_{1} & p_{2} & p_{3}\end{array}\right]^{\top}$ and $Q=\left[\begin{array}{llll}q_{0} & q_{1} & q_{2} & q_{3}\end{array}\right]^{\top}$. Then the quaternion product is written as

$$
P * Q=\left[\begin{array}{l}
p_{0} q_{0}-p_{1} q_{1}-p_{2} q_{2}-p_{3} q_{3} \\
p_{0} q_{1}+p_{1} q_{0}+p_{2} q_{3}-p_{3} q_{2} \\
p_{0} q_{2}+p_{2} q_{0}+p_{3} q_{1}-p_{1} q_{3} \\
p_{0} q_{3}+p_{3} q_{0}+p_{1} q_{2}-p_{2} q_{1}
\end{array}\right] .
$$


The quaternion product of two unit quaternions is a unit quaternion. By the definition of the quaternion the quaternions $Q$ and $-Q$ produce the same rotation. This is referred to as the dual covering. The quaternion identity is given by $Q_{I}=\left[\begin{array}{llll}1 & 0 & 0 & 0\end{array}\right]^{\top}$.

A pure quaternion is a quaternion with zero scalar part. Any vector, $\overline{\boldsymbol{v}}=\left[\begin{array}{lll}x & y & z\end{array}\right]^{\top}$ can be represented by a pure quaternion

$$
\boldsymbol{v}=\left[\begin{array}{l}
0 \\
\overline{\boldsymbol{v}}
\end{array}\right] .
$$

The conjugate of a quaternion is defined as $Q^{*}=$ $\left[\begin{array}{llll}q_{0} & -q_{1} & -q_{2} & -q_{3}\end{array}\right]^{\top}$.

\section{B. Quaternions and Rotations}

Let a vector, $\overline{\boldsymbol{v}}_{1}$, be represented by the pure quaternion $\boldsymbol{v}_{1}$. This vector can be rotated $\phi$ radians around the axis $\boldsymbol{n}$ by

$$
\boldsymbol{v}_{2}=Q * \boldsymbol{v}_{1} * Q^{*}
$$

Every vector $\overline{\boldsymbol{v}} \in \mathbb{R}^{3}$ can be represented by a pure quaternion, hence $\boldsymbol{v}$ is not necessarily a unit quaternion. The quaternion, $Q(\phi, \boldsymbol{n})$, however, is unitary. This represents the angle and the axis that the vector $\overline{\boldsymbol{v}}_{1}$ is to be rotated about. The resulting vector, $\overline{\boldsymbol{v}}_{2}$, is then of the same length as $\overline{\boldsymbol{v}}_{1}$ if and only if $Q$ is a unit quaternion. The quaternion representation also leads to a useful formula for finding the shortest rotation from one orientation to another. Let $P$ and $Q$ be two orientations. Then, by taking

$$
E=P^{*} * Q,
$$

$E$ will rotate $P$ into $Q$ by the shortest rotation.

Note that equation (7) rotates one frame into another frame. By a frame it is meant a coordinate system in $\mathbb{R}^{3}$ using Cartesian coordinates. One frame with respect to another frame represents three degrees of freedom and is referred to as an attitude orientation. Equation (6) rotates one vector into another vector and has two degrees of freedom (e.g. longitude and latitude) [9]. A unit vector with respect to a unit reference vector is referred to as an attitude direction. Henceforth, when referred to direction, this is the direction of the $z$-axis of the body frame with respect to the $z$-axis of the reference frame.

\section{QUATERNION VOLUMES}

\section{A. General Definition}

A set of frames that correspond to a reference frame by a rotation about a fixed axis, $\boldsymbol{n}$, can be represented by a quaternion and some restriction ${ }^{1}$

$$
Q(\phi, \boldsymbol{n}), \quad \text { for } \phi_{\min }<\phi<\phi_{\max } .
$$

When restrictions are not limited to one axis only, a more general description of all allowed orientations can be defined

\footnotetext{
${ }^{1}$ The dual covering allows every rotation to be described twice. In this paper, however, it is only described once, so that all angles are assumed to be in the interval $[-\pi, \pi]$. It is also assumed that all angles of inverse trigonometric functions are in this interval with the correct sign. For arctan, this is denoted $\arctan 2$.
}

by a combination of rotations given by the quaternion product of two or more quaternions and their restrictions. In this paper, only sets of frames that can be described by a sequence of rotations about fixed axes are treated.

Definition 3.1 (Quaternion Volume): A quaternion volume, $Q^{\otimes}$, is defined as

$Q^{\otimes} \triangleq\left\{Q\left(\phi_{1}, \ldots, \phi_{n}, \boldsymbol{n}_{1}, \ldots, \boldsymbol{n}_{n}\right) \mid \phi_{1, \min } \leq \phi_{1} \leq \phi_{1, \max }\right.$

$$
\begin{gathered}
\vdots \\
\left.\phi_{n, \min } \leq \phi_{n} \leq \phi_{n, \max }\right\}
\end{gathered}
$$

for $n \geq 1$ and where

$Q\left(\phi_{1}, \ldots, \phi_{n}, \boldsymbol{n}_{1}, \ldots, \boldsymbol{n}_{n}\right)=Q\left(\phi_{1}, \boldsymbol{n}_{1}\right) * \cdots * Q\left(\phi_{n}, \boldsymbol{n}_{n}\right)$.

From the above it is clear that a quaternion volume is obtained by the quaternion product of one or more quaternion volumes. This is stated in the next proposition.

Proposition 3.1 (Quaternion Product of Quat. Volume(s)): The quaternion product of two quaternion volumes, or a quaternion volume and a quaternion, is itself a quaternion volume.

Proof: By equation (3) the quaternion product of two quaternions is a quaternion. Let $P$ be a quaternion with the restrictions $\phi_{\min } \leq \phi \leq \phi_{\max }$. Then it is a quaternion volume by definition 3.1 with $n=1$. Then the quaternion product $E=P * Q$ consists of the 16 elements of equation (4). Let $Q$ be a quaternion, then $E$ can be written in terms of $e_{0-3}$.

$$
\begin{aligned}
& e_{0}=p_{0}(\phi) q_{0}-p_{1}(\phi) q_{1}-p_{2}(\phi) q_{2}-p_{3}(\phi) q_{3}, \\
& e_{1}=p_{0}(\phi) q_{1}+p_{1}(\phi) q_{0}+p_{2}(\phi) q_{3}-p_{3}(\phi) q_{2}, \\
& e_{2}=p_{0}(\phi) q_{2}+p_{2}(\phi) q_{0}+p_{3}(\phi) q_{1}-p_{1}(\phi) q_{3}, \\
& e_{3}=p_{0}(\phi) q_{3}+p_{3}(\phi) q_{0}+p_{1}(\phi) q_{2}-p_{2}(\phi) q_{1} .
\end{aligned}
$$

Note that, as $Q$ is a quaternion, the elements of $E$ are sums of the products of a constant $\left(q_{0-3}\right)$ and the elements of the quaternion volume $\left(p_{0-3}(\phi)\right)$. By representing $P \in \mathbb{R}^{4}$ and $Q \in \mathbb{R}^{4}$ as four-tuples, the quaternion product is given by (11)-(14) and the field property closure $^{2}$ is satisfied so that $e_{0-3} \in \mathbb{R}$. Thus, $e_{0-3}$ are functions of $\phi$ so that the restrictions on $\phi$ can be applied to the quaternion product. Furthermore, as $\|P\|=1$ for all $\phi,\|E\|=1$ so that $E$ is a quaternion volume by definition 3.1 with $n=2$.

Similarly, when both $P$ and $Q$ are quaternion volumes the elements of $E$ are sums of products of $p_{i}\left(\phi_{1}\right) q_{j}\left(\phi_{2}\right)$ and $E$ is a quaternion volume by the same argumentation. The same argumentation applies when $P$ and $Q$ are quaternions or quaternion volumes with more than one restriction, $n>1$.

\section{B. Quaternion Volumes by Rotations Sequences}

A rotation sequence describes a rotation about one coordinate axis followed by a rotation about another of the coordinate axes in the rotated coordinate system. A general framework on how to construct easily visualisable quaternion

\footnotetext{
${ }^{2}$ The real numbers are closed under addition and multiplication, hence if $\mathrm{a}$ and $\mathrm{b}$ are real numbers, so are $\mathrm{a}+\mathrm{b}$ and $\mathrm{ab}$ [7].
} 
volumes by rotation sequences is presented. The rotation sequence starts with two subsequent rotations about two coordinate axes, represented by the quaternion $Q_{s}$. This defines the attitude direction. The last degree of freedom is added by a rotation about the direction vector, here the $z$-axis, by $Q_{z}$. In equation (6), let $Q_{z}$ represent the vector to be rotated and let $Q_{s}$ be the quaternion describing the direction of this vector. Then the rotation sequence

$$
\mathcal{V}=Q_{s} * Q_{z} * Q_{s}^{*}
$$

represents the direction of the $z$-axis for a given rotation $Q_{s}$ given by the direction of the vector part of $\mathcal{V}$ and the rotation about the $z$-axis given by the scalar part or length of the vector part of $\mathcal{V}$ by $\psi=2 \arcsin (\|\overline{\boldsymbol{v}}\|)=2 \arccos \left(v_{0}\right) \operatorname{sgn}(\psi)$. Henceforth, $\mathcal{V}$ is called a visualising quaternion. Note that $\mathcal{V}$ does not represent a rotation. It is used as a tool to visualise rotations and as a help to define an appropriate set of frames for different applications. The visualising quaternion and the corresponding quaternion should be viewed upon as a pair, $(Q, \mathcal{V})$, where the visualising quaternion, $\mathcal{V}$, gives an intuitive description of a rotation of a frame by $Q$.

Let the vector part of the visualising quaternion be plotted as a point in the $x y z$-sphere. Then the direction of the $z$ axis, rotated by the corresponding quaternion is given by the vector from the origin to this point, and the rotation about the $z$-axis itself is given by the length of this vector. Hence, a continuous set of quaternions (a quaternion volume) is represented by a "cloud" in the $x y z$-sphere describing the corresponding set of orientations.

The quaternion that rotates the reference frame into the orientation described by equation (15) is then given by

$$
Q=Q_{s} * Q_{z}
$$

Finally, the quaternion volume is given by restricting the allowed rotations of each quaternion.

Given a visualising quaternion volume by the sequence

$$
\mathcal{V}^{\otimes}=Q_{s}^{\otimes} * Q_{z}^{\otimes} *\left(Q_{s}^{\otimes}\right)^{*}
$$

and the restrictions on $Q_{s}^{\otimes}$ and $Q_{z}^{\otimes}$. Then the corresponding quaternion volume that results in the set of orientations described by $\mathcal{V}^{\otimes}$ is given by

$$
Q^{\otimes}=Q_{s}^{\otimes} * Q_{z}^{\otimes}
$$

with the same restrictions applied to $Q^{\otimes}$ as to $\mathcal{V}^{\otimes}$.

Figure 1 shows the difference between the quaternion volume and the visualising quaternion volume when the vector part is plotted in the $x y z$-sphere ${ }^{3}$.

Note that the dual covering also applies to the visualising quaternion volume. Hence, one should always keep track of the sign of the rotation so that a negative rotation about the $z$-axis is not interpreted as an opposite direction of the $z$ axis. This can be done by moving the negative sign to the scalar part (which is positive in $(-\pi, \pi)$ or to assume all angles in the interval $(0,2 \pi)$ where $\sin \left(\frac{\phi}{2}\right)$ is positive.

\footnotetext{
${ }^{3}$ In figure 1 , the orientations are plotted by $\sin \left(\frac{\phi}{2}\right) n$ (the orthographic orientation map [8]) for both the quaternion volume and the visualising quaternion volume.
}
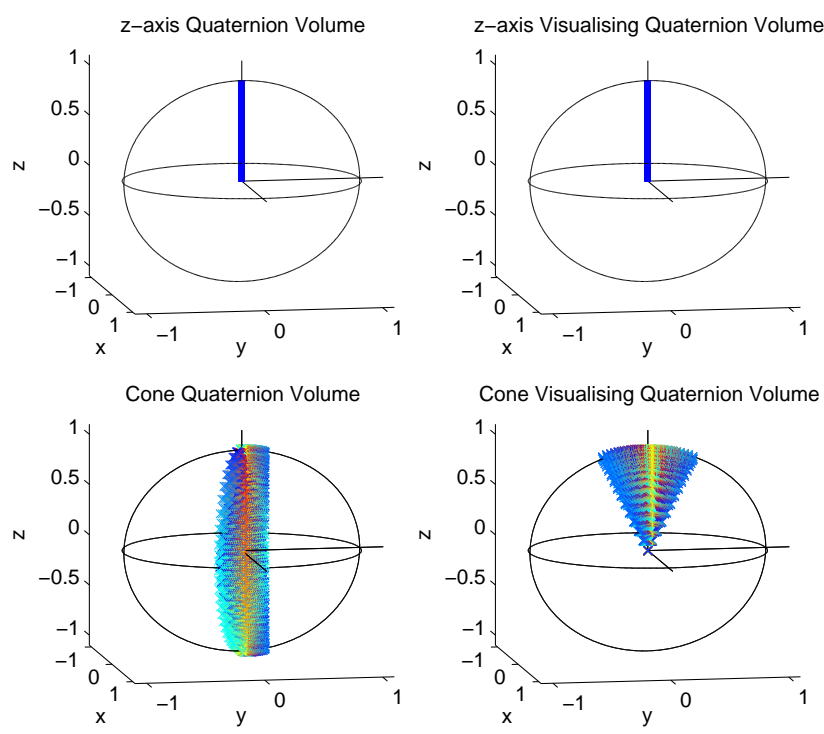

Fig. 1. The quaternion volume and the visualising quaternion volume in the $x y z$-sphere. The upper plots show a freedom about the $z$-axis and the lower plots show all vectors that span out a cone and the orientations about these vectors. The visualising quaternion volume gives a more intuitive picture of the orientations described by the quaternion volume than the quaternion volume itself when plotted in the $x y z$-sphere.

\section{Reorientation of Quaternion Volumes}

Let $Q^{\otimes}$ be a quaternion volume and the quaternion $P$ represent some transformation on $Q^{\otimes}$. It will be claimed that the transformation $Q_{P}^{\otimes}=P * Q^{\otimes}$ rotates the entire set of frames by a rotation $P$. Similarly, the transformation $Q_{P}^{\otimes}=P^{*} * Q^{\otimes}$ allows the set of frames represented by the quaternion volume to be represented with respect to a new reference frame $P$. The transformation induced by changing from one reference orientation to another is called reorientation [8].

Proposition 3.2 (Transformation of Quaternion Volumes): Any quaternion volume, $Q^{\otimes}$, represented with respect to the identity frame can be transformed into another quaternion volume by

$$
Q_{P}^{\otimes}=P * Q^{\otimes}
$$

where the orientations represented by $Q_{P}^{\otimes}$ relate to $P$ in the same way as $Q^{\otimes}$ relates to the identity frame.

Proof: The quaternion product $E=P * Q$ can be viewed upon as a rotation $P$ followed by a rotation $Q$ with respect to the new frame that resulted from the first rotation $P$. Hence, $E$ relates to $P$ in the same way as $Q$ relates to the identity frame. By the same argumentation the quaternion volume $Q_{P}^{\otimes}$ relates to $P$ in the same way as $Q^{\otimes}$ relates to the identity frame.

In proposition 3.2, the reference frame is kept constant and all the elements of the quaternion volume are rotated by $P$. Reorientation, however, is a rotation of the reference frame (change of observer) while the quaternion volume is kept constant. The proof of the reorientation $Q_{P}^{\otimes}=P^{*} * Q^{\otimes}$ is constructed in the same way as the proof of proposition 3.2 . 
Comment 1: From equations (3) and (6), four different ways of transforming quaternion volumes arise.
1) $Q_{P_{1}}^{\otimes}=Q^{\otimes} * P$
3) $Q_{P_{3}}^{\otimes}=Q^{\otimes} * P *\left(Q^{\otimes}\right)^{*}$
2) $Q_{P_{2}}^{\otimes}=P * Q^{\otimes}$
4) $Q_{P_{4}}^{\otimes}=P * Q^{\otimes} * P^{*}$

The first transformation is used in [6] to find a set of frames, all with one axis pointing in a fixed direction, as a mean to find an optimal path in the quaternion space. If $Q^{\otimes}$ represents a freedom about one of the coordinate axes, say the $z$-axis, $Q_{P_{1}}^{\otimes}$ can also represent a set of orientations where the $z$-axes have the same angles with respect to the reference frame $z$ axis, determined by $P$. For this special case, the same result is obtained by the third representation. Even though the two representations present the same set of vectors they differ in orientation. The set of frames described by $Q_{P_{1}}^{\otimes}$ is the set that results from rotating the frame represented by $P$ about the coordinate axis, while $Q_{P_{3}}^{\otimes}$ is the set of frames when the shortest rotation is taken from the reference frame to the directions described by $P$ and $Q^{\otimes}$.

\section{CoOrdinate Axis Rotation}

There are several ways of representing the proximity of two frames [3], [5]. Here, the proximity of two frames will be described by the rotation required to take one frame into the other by the shortest rotation.

Definition 4.1 (Quaternion Space Proximity): Given two orientations represented by the two quaternions $P$ and $Q$. Let the error quaternion be denoted

$$
E=P^{*} * Q
$$

Then the scalar part of $E, e_{0}$, describes the proximity of the two frames.

Definition 4.2 (Minimal Rotation): The larger (closer to $1^{4}$ ) the error quaternion scalar part $e_{0}$, the closer are the two orientations $P$ and $Q$.

An uncountable number of devices have only one degree of rotational freedom, ranging from human elbows and revolute robotic joints to satellites with only one operating actuator. The control of a one-actuator satellite is important whenever actuator failure occurs. Two questions arise:

1) How close to the desired orientation can one get with just one degree of freedom.

2) What is the reachable orientation closest to the desired orientation.

Assume that $P_{z}^{\otimes}$ represents the set of orientations when the identity frame is rotated about the $z$-axis. Then the problem is to find the $P_{z}$ that that is closest to $Q$.

Proposition 4.1 (Optimal Rotation): Consider an orientation $Q=\left[\begin{array}{llll}q_{0} & q_{1} & q_{2} & q_{3}\end{array}\right]^{\top}$. The orientation described by the quaternion $P_{z}=\left[\begin{array}{llll}p_{0} & 0 & 0 & p_{3}\end{array}\right]^{\top}$ that is closest to $Q$ (by definition 4.1 and 4.2) is given by

$$
p_{0}=\frac{ \pm_{s} q_{0}}{\sqrt{q_{0}^{2}+q_{3}^{2}}}
$$

\footnotetext{
${ }^{4}$ Note that an equally good description of proximity is given when $e_{0}$ approaches -1 . As $\cos \left(\frac{\phi}{2}\right)$ is positive for $\phi$ in the chosen interval $(-\pi, \pi)$, the positive value of $e_{0}$ is chosen.
}

$$
p_{3}=\frac{ \pm_{s} q_{3}}{\sqrt{q_{0}^{2}+q_{3}^{2}}}
$$

where the two $\pm_{s}$ have the same sign.

Proof: $E=P^{*} * Q$ can be written

$$
\begin{aligned}
& {\left[\begin{array}{l}
e_{0} \\
e_{3}
\end{array}\right]=\left[\begin{array}{cc}
p_{0} & p_{3} \\
-p_{3} & p_{0}
\end{array}\right]\left[\begin{array}{l}
q_{0} \\
q_{3}
\end{array}\right]} \\
& {\left[\begin{array}{l}
e_{1} \\
e_{2}
\end{array}\right]=\left[\begin{array}{cc}
p_{0} & p_{3} \\
-p_{3} & p_{0}
\end{array}\right]\left[\begin{array}{l}
q_{1} \\
q_{2}
\end{array}\right]}
\end{aligned}
$$

By definitions 4.1 and 4.2, the quaternion $P_{z}$ that is closest to $Q$ is found by the error quaternion with $e_{0}$ closest to 1 .

$$
\begin{aligned}
e_{0} & =p_{0} q_{0}+p_{3} q_{3} \\
& =q_{0} \cos \left(\frac{\psi}{2}\right)+q_{3} \sin \left(\frac{\psi}{2}\right),
\end{aligned}
$$

so that

$$
\frac{\mathrm{d} e_{0}}{\mathrm{~d} \psi}=-\frac{q_{0}}{2} \sin \left(\frac{\psi}{2}\right)+\frac{q_{3}}{2} \cos \left(\frac{\psi}{2}\right) .
$$

Let $\frac{\mathrm{d} e_{0}}{\mathrm{~d} \psi}=0$. Then

$$
\tan \left(\frac{\psi}{2}\right)=\frac{q_{3}}{q_{0}} .
$$

Then by using $\arctan (x)=\arcsin \left(\frac{x}{\sqrt{1+x^{2}}}\right)[10], \psi$ is written as

$$
\begin{aligned}
\psi & =2 \arctan \left(\frac{q_{3}}{q_{0}}\right) \\
& =2 \arcsin \left(\frac{\frac{q_{3}}{q_{0}}}{\sqrt{1+\left(\frac{q_{3}}{q_{0}}\right)^{2}}}\right) \\
& =2 \arcsin \left(\frac{q_{3}}{\sqrt{q_{0}^{2}+q_{3}^{2}}}\right) .
\end{aligned}
$$

From the definition of the quaternion

$$
\psi=2 \arcsin \left(p_{3}\right) \text {. }
$$

By comparing equations (31) and (32), equation (22) is given. Similarly by $\arctan (x)=\arccos \left(\frac{1}{\sqrt{1+x^{2}}}\right) \operatorname{sgn}(x)$

$$
\begin{aligned}
\psi & =2 \arctan \left(\frac{q_{3}}{q_{0}}\right) \\
& =2 \arccos \left(\frac{1}{\sqrt{1+\left(\frac{q_{3}}{q_{0}}\right)^{2}}}\right) \operatorname{sgn}\left(\frac{q_{3}}{q_{0}}\right) \\
& =2 \arccos \left(\frac{q_{0}}{\sqrt{q_{0}^{2}+q_{3}^{2}}}\right) \operatorname{sgn}\left(\frac{q_{3}}{q_{0}}\right) .
\end{aligned}
$$

Note that the sign of $\psi=2 \arccos \left(p_{0}\right) \operatorname{sgn}(\psi)$ is given by equation (32). Hence, equation (21) is found. For $\psi$ to be in the interval $[-\pi, \pi]$, the sign $\pm_{s}$ is chosen positive, so that $e_{0}$ is positive.

Similarly when $P$ rotates about the $x$ - and $y$-axis.

The largest rotation is given when $e_{0}$ is close to zero.

$$
\begin{aligned}
e_{0} & =p_{0} q_{0}+p_{3} q_{3} \\
& =q_{0} \cos \left(\frac{\psi}{2}\right)+q_{3} \sin \left(\frac{\psi}{2}\right)=0 . \\
\tan \left(\frac{\psi}{2}\right) & =-\frac{q_{0}}{q_{3}} .
\end{aligned}
$$


Similar to the proof of proposition 4.1, the orientation $P_{z}$ furthest away from $Q$ is given by

$$
\begin{aligned}
& p_{0}=\frac{ \pm_{s} q_{3}}{\sqrt{q_{0}^{2}+q_{3}^{2}}} \\
& p_{3}=\frac{ \pm_{t} q_{0}}{\sqrt{q_{0}^{2}+q_{3}^{2}}}
\end{aligned}
$$

where the $\pm_{s}$ and $\pm_{t}$ have opposite signs.

\section{Quaternion Volume Desired Attitude}

In the following, a satellite is used to illustrate the results presented above and how they apply to the control of rigid bodies. Two basic problems are addressed in this paper.

1) Fuel consumption is critical in the control of satellites. A methodology on how to represent the desired attitude by a quaternion volume as a mean to save energy is proposed.

2) Failure in one or more of the satellite actuators greatly complicates the control and can result in a desired attitude that is out of reach. A method on how to take the satellite as close as possible to the desired attitude with just one actuator is proposed.

A satellite with three actuators is considered. Each actuator applies a torque about one of the coordinate axes of the satellite body frame.

The body frame and desired attitude are defined with respect to the North-East-Down coordinate system (NEDframe). The $x$-axis of the body frame points in the speed direction and the operating device (camera, telescope, transponder, etc) is aligned along the body frame $z$-axis.

\section{A. Desired Attitude Direction}

First assume that the satellite attitude must be aligned such that the $z$-axis is always orthogonal to the earth's surface, pointing towards the earth. This gives the satellite one degree of freedom about the $z$-axis. An arbitrary rotation, $\psi$, about the $z$-axis can be represented by the quaternion volume

$Q_{\text {free }}^{\otimes}=\left[\begin{array}{llll}\cos \left(\frac{\psi}{2}\right) & 0 & 0 & \sin \left(\frac{\psi}{2}\right)\end{array}\right]^{\top}, \quad$ for $-\pi<\psi<\pi$.

Hence, the quaternion volume is given with respect to the identity frame. Further, assume the desired quaternion volume instead is to be rotated by $Q_{d}=\left[\begin{array}{llll}d_{0} & d_{1} & d_{2} & d_{3}\end{array}\right]^{\top}$ with respect to the identity frame. The quaternion volume that describes all attitudes where the $z$-axis points in the same direction as the $z$-axis of $Q_{d}$ is given by $Q_{d}^{\otimes}=Q_{d} * Q_{\text {free }}^{\otimes}$ so that

$$
Q_{d}^{\otimes}=\left[\begin{array}{l}
d_{0} \cos \left(\frac{\psi}{2}\right)-d_{3} \sin \left(\frac{\psi}{2}\right) \\
d_{1} \cos \left(\frac{\psi}{2}\right)+d_{2} \sin \left(\frac{\psi}{2}\right) \\
d_{2} \cos \left(\frac{\psi}{2}\right)-d_{1} \sin \left(\frac{\psi}{2}\right) \\
d_{3} \cos \left(\frac{\psi}{2}\right)+d_{0} \sin \left(\frac{\psi}{2}\right)
\end{array}\right], \quad \text { for } \quad-\pi<\psi<\pi .
$$

Example 1: If the desired orientation is set so that the $z$-axis is always orthogonal to the $x y$-plane, pointing outwards into space, by a rotation about the $y$-axis, $Q_{d}=$ $\left[\begin{array}{llll}0 & 0 & 1 & 0\end{array}\right]^{\top}$, equation (42) simplifies to

$Q_{d}^{\otimes}=\left[\begin{array}{llll}0 & \sin \left(\frac{\psi}{2}\right) & \cos \left(\frac{\psi}{2}\right) & 0\end{array}\right]^{\top}, \quad$ for $-\pi<\psi<\pi$.

It can be shown that this quaternion volume represents all attitudes with a $z$-axis in the opposite direction of the NED-frame $z$-axis.

$$
\begin{aligned}
\boldsymbol{z}^{\otimes} & =Q_{d}^{\otimes} * \boldsymbol{v}_{z} *\left(Q_{d}^{\otimes}\right)^{*} \\
& =\left[\begin{array}{c}
0 \\
\sin \left(\frac{\psi}{2}\right) \\
\cos \left(\frac{\psi}{2}\right) \\
0
\end{array}\right] *\left[\begin{array}{l}
0 \\
0 \\
0 \\
1
\end{array}\right] *\left[\begin{array}{c}
0 \\
-\sin \left(\frac{\psi}{2}\right) \\
-\cos \left(\frac{\psi}{2}\right) \\
0
\end{array}\right] \\
& =\left[\begin{array}{c}
-\cos \left(\frac{\psi}{2}\right) \sin \left(\frac{\psi}{2}\right)+\cos \left(\frac{\psi}{2}\right) \sin \left(\frac{\psi}{2}\right) \\
0 \\
0
\end{array}\right] \\
& =\left[\begin{array}{c}
0 \\
0 \\
0 \\
0 \\
-1
\end{array}\right], \quad \text { for }-\pi<\psi<\pi .
\end{aligned}
$$

\section{B. Desired Attitude Orientation}

The attitude can be represented as a set of frames. This set can be composed by a rotation sequence of quaternion volumes. Two rotation sequences are discussed, the ZYZYZsequence, as in [6] and [8], and the XYZYX-sequence.

1) The ZYZYZ-sequence: The ZYZYZ-sequence allows the desired attitude to be defined as a set of vectors that span out a cone about the reference $z$-axis and all orientations about these vectors. Let $Q_{s}(\alpha, \beta)=Q(\alpha, \boldsymbol{z}) * Q(\beta, \boldsymbol{y})$ where $Q(\alpha, \boldsymbol{z})=\left[\begin{array}{llll}\cos \left(\frac{\alpha}{2}\right) & 0 & 0 & \sin \left(\frac{\alpha}{2}\right)\end{array}\right]^{\top}$ and $Q(\beta, \boldsymbol{y})=$ $\left[\begin{array}{llll}\cos \left(\frac{\beta}{2}\right) & 0 & \sin \left(\frac{\beta}{2}\right) & 0\end{array}\right]^{\top}$ so that

$$
Q_{s}(\alpha, \beta)=\left[\begin{array}{c}
\cos \left(\frac{\alpha}{2}\right) \cos \left(\frac{\beta}{2}\right) \\
-\sin \left(\frac{\alpha}{2}\right) \sin \left(\frac{\beta}{2}\right) \\
\cos \left(\frac{\alpha}{2}\right) \sin \left(\frac{\beta}{2}\right) \\
\sin \left(\frac{\alpha}{2}\right) \cos \left(\frac{\beta}{2}\right)
\end{array}\right] \text {. }
$$

The quaternion volume can be visualised in the $x y z$-sphere (see figure 1) by the three last elements of

$$
\mathcal{V}^{\otimes}(\alpha, \beta, \gamma)=\left[\begin{array}{c}
\cos \left(\frac{\gamma}{2}\right) \\
\sin \left(\frac{\gamma}{2}\right) \sin (\beta) \cos (\alpha) \\
\sin \left(\frac{\gamma}{2}\right) \sin (\alpha) \sin (\beta) \\
\sin \left(\frac{\gamma}{2}\right) \cos (\beta)
\end{array}\right] .
$$

$\alpha$ represents the allowed orientations about the $z$-axis of the first rotation while $\beta$ is the allowed orientation about the new $y$-axis. If $\alpha$ has no restrictions, $\beta$ is the offset angle that defines a cone with the $z$-axis at the centre. $\gamma$ restricts the orientation about the $z$-axis itself. A cone sector that allows a deviation, $b_{\max }$, in the sector defined by the restrictions on $\alpha$ in the $x y$-plane is defined by

$$
Q_{d}^{\otimes}=Q_{s}^{\otimes} * Q_{z}^{\otimes}=\left[\begin{array}{l}
\cos \left(\frac{\beta}{2}\right) \cos \left(\frac{\gamma}{2}+\frac{\alpha}{2}\right) \\
\sin \left(\frac{\beta}{2}\right) \sin \left(\frac{\gamma}{2}-\frac{\alpha}{2}\right) \\
\sin \left(\frac{\beta}{2}\right) \cos \left(\frac{\gamma}{2}-\frac{\alpha}{2}\right) \\
\cos \left(\frac{\beta}{2}\right) \sin \left(\frac{\gamma}{2}+\frac{\alpha}{2}\right)
\end{array}\right]
$$


and the restrictions

$$
\begin{gathered}
a_{\min } \leq \alpha \leq a_{\max } \\
0 \leq \beta \leq b_{\max } \\
c_{\min } \leq \gamma \leq c_{\max }
\end{gathered}
$$

Example 2: Assume a satellite where the $z$-axis is to point outwards into space. Further assume that a small error, $b_{\max }$, in the orientation is allowed and only the attitude directions are restricted. The set of frames describing these attitudes is given by (48) and the restrictions

$$
\begin{gathered}
-\pi \leq \alpha \leq \pi \\
\pi \leq \beta \leq \pi+b_{\max } \\
-\pi \leq \gamma \leq \pi
\end{gathered}
$$

It can be showed that this is the same as substituting $\beta \leftarrow \pi+\beta$ and $\alpha \leftarrow-\alpha$ into equation (48) so that

$$
Q_{d}^{\otimes}=Q_{s}^{\otimes} * Q_{z}^{\otimes}=\left[\begin{array}{c}
-\sin \left(\frac{\beta}{2}\right) \cos \left(\frac{\gamma}{2}-\frac{\alpha}{2}\right) \\
\cos \left(\frac{\beta}{2}\right) \sin \left(\frac{\gamma}{2}+\frac{\alpha}{2}\right) \\
\cos \left(\frac{\beta}{2}\right) \cos \left(\frac{\gamma}{2}+\frac{\alpha}{2}\right) \\
-\sin \left(\frac{\beta}{2}\right) \sin \left(\frac{\gamma}{2}-\frac{\alpha}{2}\right)
\end{array}\right]
$$

and keeping the restrictions (49)-(51). Note that equation (55) can also be obtained by rotating the quaternion volume of the previous example by $\pi$ radians about the $y$ axis, hence by equation (19) with $P=\left[\begin{array}{llll}0 & 0 & 1 & 0\end{array}\right]^{\top}$ and $Q^{\otimes}=\left[\begin{array}{llll}q_{0} & q_{1} & q_{2} & q_{3}\end{array}\right]^{\top}$ as in (48) so that $Q_{d}^{\otimes}=$ $\left[\begin{array}{llll}-q_{2} & q_{3} & q_{0} & -q_{1}\end{array}\right]^{\top}$, which is the same as equation (55).

2) The $X Y Z Y X$-sequence: The XYZYX-sequence defines a pyramid of allowed orientations where the allowed orientations about the $x$-axis and the (new) $y$-axis are restricted. This is a good estimation of restricting the orientation about the globally defined $x$ - and $y$-axes whenever the angles are kept small. $Q_{s}(\alpha, \beta)$ is then given by

$$
Q_{s}(\alpha, \beta)=\left[\begin{array}{c}
\cos \left(\frac{\alpha}{2}\right) \cos \left(\frac{\beta}{2}\right) \\
\sin \left(\frac{\alpha}{2}\right) \cos \left(\frac{\beta}{2}\right) \\
\cos \left(\frac{\alpha}{2}\right) \sin \left(\frac{\beta}{2}\right) \\
\sin \left(\frac{\alpha}{2}\right) \sin \left(\frac{\beta}{2}\right)
\end{array}\right],
$$

and visualised by

$$
\mathcal{V}^{\otimes}(\alpha, \beta, \gamma)=\left[\begin{array}{c}
\cos \left(\frac{\gamma}{2}\right) \\
\sin \left(\frac{\gamma}{2}\right) \sin (\beta) \\
-\sin \left(\frac{\gamma}{2}\right) \sin (\alpha) \cos (\beta) \\
\sin \left(\frac{\gamma}{2}\right) \cos (\alpha) \cos (\beta)
\end{array}\right] .
$$

The corresponding quaternion volume is again given by

$$
Q_{d}^{\otimes}=Q_{s}^{\otimes} * Q_{z}^{\otimes}
$$

and the restrictions

$$
\begin{aligned}
& a_{\text {min }} \leq \alpha \leq a_{\max } \\
& b_{\min } \leq \beta \leq b_{\max } \\
& c_{\text {min }} \leq \gamma \leq c_{\max }
\end{aligned}
$$

Example 3: Assume a satellite where the attitude is to be restricted similarly to example 2, but instead of allowing some error in the orientation, an orientation error about the $x$ - and $y$-axes are restricted to $\pm a$ and $\pm b$. Then the set of frames describing these attitudes is given by (58) and the restrictions

$$
\begin{gathered}
\pi-a \leq \alpha \leq \pi+a \\
-b \leq \beta \leq b \\
-\pi \leq \gamma \leq \pi
\end{gathered}
$$

\section{Control}

Two ways to exploit the quaternion volume representation to reduce fuel consumption are presented.

1) Let the desired attitude (one frame only) take part in a control loop. When the attitude is inside the attitude specifications given by the quaternion volume, some action is taken to save energy. This may be to switch to another controller which requires less energy or to switch to another desired attitude inside the quaternion volume, closer or equal to the current attitude. Note also that if the quaternion volume defines a set of orientations close to some reference orientation, a linearised model of the satellite may be used.

2) Find the frame within the set of frames restricted by the quaternion volume that corresponds to the shortest rotation from the current orientation and set this as the desired attitude.

Two problems arise.

1) A test to verify if a frame is inside the quaternion volume is needed.

2) Find the orientation inside the quaternion volume that results in the shortest rotation from the current orientation.

\section{A. Quaternion Volume Test}

Consider a quaternion volume defined by the ZYZYZsequence. A test to verify if a query quaternion $Q_{q r y}=$ $\left[\begin{array}{llll}q_{0} & q_{1} & q_{2} & q_{3}\end{array}\right]^{\top}$ is an element of the quaternion volume is presented.

A query quaternion can be represented in terms of $\alpha, \beta$ and $\gamma$. The transformation between the quaternion representation and the $(\alpha, \beta, \gamma)$-representation can be performed in many ways, by geometric analysis, by the visualising quaternion or through a quaternion/orientation map. The first method is often the easiest and most intuitive method and works well when only the direction is concerned. When the full orientation is to be determined, this approach is not suitable. In the following, it is shown how this method can be combined with the visualising quaternion to find the orientation.

By noting that $\alpha$ and $\beta$ can be seen from the direction of the $z$-axis only (not from the entire frame), they can be found from the vector $\bar{z}=\left[\begin{array}{lll}x & y & z\end{array}\right]^{\top}$ of the rotation of the vector along the $z$-axis, $\overline{\boldsymbol{v}}_{z}=\left[\begin{array}{lll}0 & 0 & 1\end{array}\right]^{\top}$ by $\boldsymbol{z}=Q_{q r y} * \boldsymbol{v}_{z} * Q_{q r y}^{*}$. Then, by standard geometrical relations $\alpha$ and $\beta$ are found.

$$
\begin{gathered}
\alpha=\arctan 2\left(\frac{y}{x}\right), \\
\beta=\arccos (z),
\end{gathered}
$$


where $x, y$ and $z$ are the elements of $\bar{z}$ given by

$$
\overline{\boldsymbol{z}}=\left[\begin{array}{c}
2 q_{0} q_{2}+2 q_{1} q_{3} \\
2 q_{2} q_{3}-2 q_{0} q_{1} \\
q_{0}^{2}-q_{1}^{2}-q_{2}^{2}+q_{3}^{2}
\end{array}\right] .
$$

As already stated, the rotation about the $z$-axis cannot be seen from the vector rotation of the $z$-axis, but is found from $\mathcal{V}$ by $\gamma=2 \arccos \left(v_{0}\right)$. The sign of $\gamma$ is lost in the transformation but can be found by, for example the sign of the fourth element of $\mathcal{V}$. As $\alpha$ and $\beta$ are known, $\mathcal{V}$ can be written as

$$
\mathcal{V}=Q_{q r y} * Q_{s}^{*}(\alpha, \beta) .
$$

Given a query quaternion $Q_{q r y}$. Then $\alpha, \beta$ and $\gamma$ from the ZYZYZ-sequence are found by

$$
\begin{gathered}
\alpha=\arctan 2\left(\frac{q_{2} q_{3}-q_{0} q_{1}}{q_{0} q_{2}+q_{1} q_{3}}\right), \\
\beta=\arccos \left(q_{0}^{2}-q_{1}^{2}-q_{2}^{2}+q_{3}^{2}\right), \\
\gamma=2 \arccos \left(v_{0}\right) \operatorname{sgn}\left(v_{4}\right) .
\end{gathered}
$$

It is now straight forward to verify if the quaternion is inside the quaternion volume.

The quaternion volume is not always of such a structure that it can be analysed geometrically. Then the analytic expression of the quaternion volume can be used. For comparison, this approach is also shown for the ZYZYZsequence.

The quaternion volume is given by equation (48) and its restrictions.

$$
\left[\begin{array}{c}
\cos \left(\frac{\beta}{2}\right) \cos \left(\frac{\gamma}{2}+\frac{\alpha}{2}\right) \\
\sin \left(\frac{\beta}{2}\right) \sin \left(\frac{\gamma}{2}-\frac{\alpha}{2}\right) \\
\sin \left(\frac{\beta}{2}\right) \cos \left(\frac{\gamma}{2}-\frac{\alpha}{2}\right) \\
\cos \left(\frac{\beta}{2}\right) \sin \left(\frac{\gamma}{2}+\frac{\alpha}{2}\right)
\end{array}\right]=\left[\begin{array}{cc}
q_{0} \\
q_{1} \\
q_{2} \\
(I) \\
(I I I) \\
q_{3}
\end{array}\right](I V)
$$

By substituting (II) into (III), (III) simplifies to

$$
\sin \left(\frac{\beta}{2}\right) \sqrt{1-\frac{q_{1}^{2}}{\sin ^{2}\left(\frac{\beta}{2}\right)}}=q_{2}
$$

so that $\beta$ is found by

$$
\beta=2 \arcsin \sqrt{q_{1}^{2}+q_{2}^{2}} .
$$

$\beta$ is positive by definition. $\alpha$ and $\gamma$ are found by dividing (II) by (III) and (IV) by (I):

$$
\begin{aligned}
& \tan \left(\frac{\gamma}{2}-\frac{\alpha}{2}\right)=\frac{q_{1}}{q_{2}}, \\
& \tan \left(\frac{\gamma}{2}+\frac{\alpha}{2}\right)=\frac{q_{3}}{q_{0}} .
\end{aligned}
$$

Further let

$$
\begin{aligned}
& \frac{\gamma}{2}-\frac{\alpha}{2}=\arctan \left(\frac{q_{1}}{q_{2}}\right), \\
& \frac{\gamma}{2}+\frac{\alpha}{2}=\arctan \left(\frac{q_{3}}{q_{0}}\right) .
\end{aligned}
$$

so that $\alpha$ and $\gamma$ are given by

$$
\begin{aligned}
& \alpha=\arctan \left(\frac{q_{3}}{q_{0}}\right)-\arctan \left(\frac{q_{1}}{q_{2}}\right), \\
& \gamma=\arctan \left(\frac{q_{3}}{q_{0}}\right)+\arctan \left(\frac{q_{1}}{q_{2}}\right) .
\end{aligned}
$$

Hence, $\alpha$ and $\beta$ can by found by geometrical interpretation while $\alpha, \beta$ and $\gamma$ are found from the analytical expression of the quaternion volume. In the following, it is shown that these two approaches give the same result. From equation (70), $\beta$ is simplified by

$$
\begin{aligned}
\beta & =\arccos \left(q_{0}^{2}-q_{1}^{2}-q_{2}^{2}+q_{3}^{2}\right) \\
& =\arccos \left(2\left(q_{0}^{2}+q_{3}^{2}\right)-1\right)
\end{aligned}
$$

and the trigonometric relation $2 \arccos (x)=\arccos \left(2 x^{2}-1\right)$ [10] so that

$$
\beta=2 \arccos \sqrt{q_{0}^{2}+q_{3}^{2}} .
$$

By $\arccos (x)=\arcsin \left(\sqrt{1-x^{2}}\right)$ this is equal to equation (74). By $\arctan (x)+\arctan (y)=\arctan 2\left(\frac{x+y}{1-x y}\right)$, equation (79) can be written as

$$
\begin{aligned}
\alpha & =\arctan \left(\frac{q_{3}}{q_{0}}\right)-\arctan \left(\frac{q_{1}}{q_{2}}\right) \\
& =\arctan 2\left(\frac{\frac{q_{3}}{q_{0}}-\frac{q_{1}}{q_{2}}}{1+\frac{q_{1} q_{3}}{q_{0} q_{2}}}\right) \\
& =\arctan 2\left(\frac{q_{2} q_{3}-q_{0} q_{1}}{q_{0} q_{2}+q_{1} q_{3}}\right) .
\end{aligned}
$$

$\gamma$ can be written in the same way so that, alternatively, a complete description of the query quaternion can be given by

Given a query quaternion $Q_{q r y}$. Then $\alpha, \beta$ and $\gamma$ from the ZYZYZ-sequence are found by

$$
\begin{gathered}
\alpha=\arctan 2\left(\frac{q_{2} q_{3}-q_{0} q_{1}}{q_{0} q_{2}+q_{1} q_{3}}\right), \\
\beta=2 \arccos \sqrt{q_{0}^{2}+q_{3}^{2}}, \\
\gamma=\arctan 2\left(\frac{q_{2} q_{3}+q_{0} q_{1}}{q_{0} q_{2}-q_{1} q_{3}}\right) .
\end{gathered}
$$

\section{B. Transformed Quaternion Volumes}

The easiest way to verify if a query quaternion is inside a quaternion volume transformed by equation (19) is to transform the query quaternion by the opposite transformation, $P$, so that both the quaternion volume and the query quaternion are presented in the identity frame. Hence, the two problems below are identical.

$$
\begin{array}{rr}
Q_{q r y} \in P * Q^{\otimes} & ? \\
P^{*} * Q_{q r y} \in Q^{\otimes} & ?
\end{array}
$$

This operation is computationally demanding. As equation (19) gives an analytical expression of the transformed quaternion volume, the orientation should be found by a set of parameters similar to the ones found in equations (87)-(89). This may be done when the quaternion volume is on a simple form, for example by equation (55). Then the query quaternion may be tested against the restrictions in (49)-(51) directly. By following the mathematics of equations (72)(89), $\alpha, \beta$ and $\gamma$ are found with respect to the coordinate 
system of $P=\left[\begin{array}{llll}0 & 0 & 1 & 0\end{array}\right]^{\top}$ by

$$
\begin{gathered}
\alpha_{P}=\arctan 2\left(\frac{q_{0} q_{1}-q_{2} q_{3}}{q_{0} q_{2}+q_{1} q_{3}}\right), \\
\beta_{P}=2 \arcsin \sqrt{q_{0}^{2}+q_{3}^{2}}, \\
\gamma_{P}=\arctan 2\left(\frac{q_{0} q_{1}+q_{2} q_{3}}{q_{0} q_{2}-q_{1} q_{3}}\right) .
\end{gathered}
$$

Hence, as expected

$$
\begin{gathered}
\beta_{P}=\beta-\pi, \\
\alpha_{P}=-\alpha, \\
\gamma_{P}=\gamma
\end{gathered}
$$

\section{Clamping}

If any of the restrictions are violated, the quaternion might be clamped into the set of frames restricted by the quaternion volume in many ways. [6] suggests finding the nearest point in the quaternion metric. Another intuitively tempting approach is to set the exceeded value to the maximum allowed value. Then a quaternion that is inside the quaternion volume may be constructed by the definition in equation (48) directly. If the quaternion volume is on a simple form, the orientation can be clamped into the quaternion volume by the shortest rotation in order to save energy.

\section{Shortest Rotation}

How to find the orientation in the quaternion volume that results in the shortest rotation from the current attitude depends on the quaternion volume. One simple solution occurs when the quaternion volume represents a freedom about one axis. Then the theory from section IV can be applied directly. Let $Q$ be the current attitude and $P_{\text {free }}^{\otimes}=$ $\left[\begin{array}{llll}\cos \left(\frac{\psi}{2}\right) & 0 & 0 & \sin \left(\frac{\psi}{2}\right)\end{array}\right]^{\top}$ represent the set of allowed attitudes, both defined in the NED-frame. Then the attitude within the quaternion volume that is closest to the current attitude is given by

$$
\begin{aligned}
& p_{0}=\frac{ \pm_{s} q_{0}}{\sqrt{q_{0}^{2}+q_{3}^{2}}} \\
& p_{3}=\frac{ \pm_{s} q_{3}}{\sqrt{q_{0}^{2}+q_{3}^{2}}}
\end{aligned}
$$

and the rotation required to take $Q$ into $P$ (the error) is given by

$$
\psi=2 \arccos \left(e_{0}\right)
$$

where $e_{0}=p_{0} q_{0}+p_{3} q_{3}$.

\section{E. Closest Orientation}

Closely related to the problem of the previous subsection is the problem of finding the optimal rotation when actuator failure occurs. When only one actuator is functional, the satellite can only rotate about one of the axes of the body frame. Let the current body orientation be described in the NED-frame by $T$. The desired orientation, $Q_{d}$, is also given in the NED-frame. Assume that the freedom, represented by a quaternion volume, $Q_{\text {free }}^{\otimes}$, is given with respect to the body frame, so that

$$
Q_{T}^{\otimes}=T * Q_{\text {free }}^{\otimes}
$$

represent all reachable orientations. Then the problem is to find the quaternion $Q \in Q_{\text {free }}^{\otimes}$ that takes the satellite as close to the desired orientation as possible. This is given by proposition 4.1 where $E$ is given by

$$
\begin{aligned}
E & =Q_{d}^{*} * Q_{T}^{\otimes} \\
& =Q_{d}^{*} * T * Q_{\text {free }}^{\otimes} .
\end{aligned}
$$

Let $T_{d}^{*}=Q_{d}^{*} * T=\left[\begin{array}{llll}q_{0} & -q_{1} & -q_{2} & -q_{3}\end{array}\right]$. Then, as only the size (not the direction) of the rotation is considered, the closest possible orientation is given by equations (21) and (22) and the rotation needed to take the satellite from the closest reachable orientation to the desired orientation (the error) is given by equation (100).

\section{CONCLUSIONS}

The unit quaternion group is used to find a general framework for representing sets of orientations. It is also shown how this set can be represented with respect to another reference frame or how to rotate this set when the reference frame is kept constant. Several examples of sets of orientations are presented and it is shown how these sets can be represented by a quaternion and some easy to visualise restrictions. A satellite is used to illustrate how to save energy by defining the desired attitude as a set of orientations. A method to verify whether a quaternion is inside a quaternion volume is also presented. It is also shown how to find the rotation that requires less energy in order to take the desired attitude into an element of the quaternion volume. Some work is done on finding the orientation closest to the desired orientation when the desired orientation is out of reach.

\section{REFERENCES}

[1] T. A. W. Dwyer, "Exact nonlinear control of large angle rotational maneuvers," IEEE Transactions on Automatic Control, vol. AC-29 no. 9, pp. 769-774, 1984.

[2] R. Kristiansen and P. J. Nicklasson, "Satellite attitude control by quaternion-based backstepping," American Control Conference, 2005.

[3] J. S. C. Yuan, "Closed-loop manipulator control using quaternion feedback," IEEE Journal of Robotics Automation, vol. 4, no. 4, pp. 434-440, 1988.

[4] B. Xian, M. D. Queiroz, D. Dawson, and I. Walker, "Task-space tracking control of robot manipulators via quaternion feedback," IEEE Transactions on Robotics and Automation, vol. 20 no. 1, 2004.

[5] J. T.-Y. Wen and K. Kreutz-Delgado, "The attitude control problem," IEEE Transactions on Automatic Control, vol. 30 no. 10, pp. 1148$1162,1991$.

[6] A. J. Hanson, Visualizing Quaternions. Morgan Kaufmann, 2006.

[7] J. B. Kuipers, Quaternions and Rotation Sequences. Princeton University Press, 2002.

[8] B. Alpern, L. Carter, M. Grayson, and C. Pelkie, "Orientation maps: Techniques for visualizing rotations (a consumers guide)," IEEE Conference on Visualization, pp. 183-188, 1993.

[9] J. M. Ahuactzin and K. K. Gupka, "The kinematic roadmap: A motion planning based global approach for inverse kinematics of redundant robots." IEEE Trans. on Robotics and Automation, vol. 15, 1999.

[10] I. N. Bronshtein, K. A. Semendyayev, G. Musiol, and H. Muehlig, Handbook of Mathematics. Springer, 2003. 